\title{
A Multi-Criteria Decision Support System for a Routing Problem in Waste Collection
}

\author{
João A. Ferreira ${ }^{(凶)}$, Miguel Costa, Anabela Tereso, and José A. Oliveira \\ University of Minho, Department of Production and Systems, Campus de Azurém, \\ 4800-058 Guimarães, Portugal \\ \{joao.aoferreira, miguelpintodacosta\}@gmail.com, \\ $\{$ anabelat, zan\}@dps. uminho.pt
}

\begin{abstract}
This work presents a decision support system for route planning of vehicles performing waste collection for recycling. We propose a prototype system that includes three modules: route optimization, waste generation prediction, and multiple-criteria decision analysis (MCDA). In this work we focus on the application of MCDA in route optimization. The structure and functioning of the DSS is also presented.

We modelled the waste collection procedure as a routing problem, more specifically as a team orienteering problem with capacity constraints and time windows. To solve the route optimization problem we developed a cellular genetic algorithm. For the MCDA module, we employed three methods: SMART, ValueFn and Analytic Hierarchy Process (AHP).

The decision support system was tested with real-world data from a waste management company that collects recyclables, and the capabilities of the system are discussed.
\end{abstract}

Keywords: Waste collection • Vehicle Routing - Team Orienteering Problem $\cdot$ Decision Support System $\cdot$ Multiple-Criteria Decision Analysis $\cdot$ Cellular Genetic Algorithm · AHP · SMART $\cdot$ ValueFn

\section{Introduction}

Over the years, decision sciences have been applied in resource management to achieve success in the business world. In order to avoid losses and increase income while depending on the use of resources, companies tend to rely on the decisions carried out by their managers. The process of decision-making often happens over the analysis of several criteria that sometimes have conflicting objectives or induce divergent results while aiming for the same goal. Those different outcomes can either complement each other or create problems while deciding the best course of action to complete a certain task or project. Common conflicting criteria are cost (or price) and quality measures (or performance indicators). Trying to achieve a good balance between important criteria implies weighing their contributions and (adverse) consequences to the final objective, and opting 
for the best alternative for that specific situation. This process requires structuring the problem in a proper way to reduce its complexity and making better decisions.

MCDA is the acronym for multiple-criteria decision analysis, which involves the application of models and methodologies to provide the decision maker (DM) with tools that enable solving decision and planning problems, although, when there are several criteria to consider, it is very difficult, if not impossible, to determine an unique best solution. To find an overall best solution, the DMs preferences need to be included in the decision process, since there are trade-offs that need to be considered, and the importance given to each criteria depends on the DM.

The dawn of computer age enabled the development of software implementing various models and methods to solve multiple-criteria decision-making problems, and there are plenty of commercially available general-purpose products, as well as more specific ones. This kind of software became extremely useful in many different management scenarios. Often, MCDA software is included in larger (software) systems called Decision Support Systems (DSS). A DSS is an information system that supports business decision-making activities, and helps making decisions at different levels: 1) management, 2) operations and 3) planning. A DSS is usually an interactive software tool that combines data from various sources and formats and presents the user (i.e. the DM) with useful information, displayed on graphical interfaces, that helps solving decision-making problems.

In this study, we focus on a real-world problem, where management issues are faced by a waste management company (WMC) that needs to pick-up recyclable materials stored along a network of collection points. In general, successful waste management highly depends on good performing logistic systems that keep track of all needed requirements and the goals/objectives to be met by the companies. Resource management (i.e. vehicles, drivers, assistants) and designing cost-efficient waste collection routes are some of the major issues a DM in a WMC has to deal with on a daily basis, and it often involves weighting the importance of several criteria. This creates different operation scenarios that need to be assessed for the DM to determine the best solution for a certain situation. Therefore, a DSS is an important tool to assist in this decision-making process. The aim of this study was to develop a DSS that includes route optimization models and employs MCDA methods to compare different operational procedures carried out by a WMC when performing waste collections.

\section{Problem Description}

The recycling of waste materials has earned great importance over the years, and today is a vital process to our survival in a clean and healthy environment, and also to move towards a more sustainable future. Regarding waste composition, in Portugal, more than $50 \%$ of municipal solid waste is composed of recyclable materials, where paper accounts for $20.3 \%$, plastics $18 \%$, glass $6 \%$, metals $5 \%$, and textiles $3.8 \%$ [19]. The collection of recyclable materials have lately become a 
fertile subject for the development of new ideas to improve resource management and global efficiency. There is also a constant need for improvements in waste management, especially in terms of resource management.

The process of collecting recyclables usually involves three main resources that need management: 1) workers, 2) vehicles and 3) time. In addition, there are elements that impose constraints to resource management such as cost limitations in fuel expenditure, avoidance of high vehicle wearing, minimum (daily) quotas of waste to collect to be met, and also certain collection performance standards that should be attained. Many improvements often occur through successful fleet management, which greatly relies on optimization procedures applied to the design of collection routes. Designing more efficient routes implies balancing the use of resources while respecting all constraints that may be imposed.

Focusing on route design, the objective is to visit a set of waste collection points using a vehicle fleet, while respecting constraints such as vehicle capacity and maximum route duration and/or length (time spent and/or travelled distance). This kind of situation can be addressed as a vehicle routing problem (VRP) [13]. Although this description fits the mentioned waste collection problem, more flexibility is needed when designing the routes. Each collection point is assigned a certain priority level to be visited and emptied, based on their waste generation rates and current filling status. While scheduling collection routes, there is a need to select which points to visit during those routes, and only a part of those points may be collected. The VRP models can be too restrictive when employed in this situation, since the premise is to visit all points in the network, regardless of its filling status, and using as many vehicles as needed to do so. So, instead of targeting all collection points, a more fitting model named team orienteering problem (TOP) [8,11], can be applied. In this context, the TOP can be described as the problem of designing routes and assign them to a limited fleet of vehicles performing collection of recyclable waste stored along a network of collection points; each collection point has a priority level; the collection routes have maximum durations and/or distances; the vehicles have capacity limits; the selection of collection points to be visited by the vehicles is made by balancing their priorities and their contributions for route duration, route distance and quantity of waste collected per vehicle. The objective is to maximize the total amount of waste collected by all routes while respecting the time and/or distance constraints, and also capacity constraints or even time windows.

In Portugal, a major source of potentially recyclable materials is household packaging waste (HPW), which is usually composed of materials made of glass, paper/cardboard, plastic or metal. HPW is separated by citizens at the local recycling site (collection point), named ecopoint (ecological point). Given the goals Portugal has to fulfil for the recycling and recovery of HPW, there is a permanent need for increased efficiency in waste collection performed by waste management companies. Therefore, the main goal of this study is to explore new solutions and management options for a real problem faced by a Portuguese inter-municipal waste management company (WMC) that takes action across six 
municipalities and currently operates a network of more than 1,200 ecopoints. This WMC's area of operations is a mix of urban and rural areas, which prompts the demand of different strategies for waste management.

There is no requirement for the mentioned WMC to visit all their ecopoint sites every workday, as it would be unprofitable and inefficient, and of course impossible, since there is a limited vehicle fleet available. Therefore, it is necessary to select a subset of ecopoints to visit each day. Furthermore, given a planning horizon of, for example, a week, or a month, the WMC must decide, based on the priority levels of ecopoints, which ones must be visited, which ones can be visited, and which ones can be skipped during the collection routes, and then design effective routes to perform the collection of HPW. Therefore, modelling this collection procedure as a TOP is suitable. In fact, this routing problem can be modelled as a capacitated team orienteering problem with time windows (CTOPTW), due to capacity constraints on the vehicles, and because there is a time interval specified for each ecopoint, during which the waste containers must be emptied.

It is not uncommon for performance requirements in waste collection to change while aiming to accomplish different goals. There are times when a DM might find himself in difficulty to choose the best strategy for a given situation, and when performance indicators (PIs) are presented, conflicting objectives can arise. For example, the company aims to collect as much quantities of waste per route as possible, but also needs to minimize the total distance travelled by the collection vehicles. Handling these situations and deciding on what the most suitable solution is in order to attend acceptable values for the PIs, can be a time-consuming task, and so, a valuable tool to use is a DSS with MCDA capabilities, which can process a great amount of information and present solutions to the DM at a faster pace. Nonetheless, the DM assumes a central role during decision-making processes in a DSS.

The work presented in this paper is integrated in a $R \& D$ project named Genetic Algorithm for Team Orienteering Problem (GATOP). The main goal of GATOP is the development of more complete and efficient solutions for several real-life multi-level vehicle routing problems, with an emphasis on waste collection management. In this work, we intend to present a prototype DSS for management of HPW collection, and it shall be composed of different modules or elements. Our purpose is to design the proper functioning of the DSS by determining how the modules interact, how information flows between them, and how the information is processed and solutions are presented to the users. In addition, we needed to do some improvements and changes to the route optimization module for it to be able to handle different objective functions, which were formulated based on the study of real case scenarios faced by a WMC. Briefly, the tasks for this study were:

- Define alternative objectives for route design in waste collection for recycling;

- Develop a solution method to solve different objective functions for the routing problem;

- Define the set of criteria that will be used in the MCDA module; 
- Develop and implement the MCDA module;

- Define the structure and way of operation of the DSS prototype;

- Test the DSS for real case scenarios and evaluate its functionality.

\section{$3 \quad$ Literature Review}

\subsection{Solution Methods for the TOP and Other Variants}

In the context of fleet management there is a high demand for more efficient procedures and techniques to perform route planning and design. There are plenty of problems involving transportation of people and/or items/commodities that need route optimization for various purposes. This kind of problems can be addressed as a vehicle routing problem (VRP). As we stated previously, the VRP model is too restrictive for the collection of recyclable waste, and more flexibility is required to enable the selection of locations to visit. Such flexibility is achievable by modelling the collection process as a team orienteering problem (TOP). A wide variety of algorithms have been developed to solve the TOP, and some successful ones are tabu search [4,35], branch and price [7], guided local search [43], path-relinking [33], ant colony optimization [22], memetic algorithm [6], particle swarm optimization [12,32]. For the TOP with time windows (TOPTW), efficient algorithms are iterated local search [42], variable local search, ant colony system [25], variable neighbourhood search [24,40], and hybrid iterated local search [34]. Other variant of the TOP includes capacity constraints (CTOP) has been receiving attention lately, with tabu search being a good solution option [2,3]. Other algorithms followed, as well as new variants of the TOP and CTOP. Promising algorithms based on other methodologies are also found in the literature of routing problems, such as genetic algorithms $[15,16,27,28,36,37]$ and cellular genetic algorithms [1].

\subsection{Application of Multi-Criteria Decision Analysis to Routing Problems}

After a wide range review of literature on the application of multi-criteria decision analysis in decision support systems for routing problems over the last decade, the authors came across some interesting works in this research field.

In 2002, the author Jacek Zak [45] brought attention to several problems faced by many transportation companies. One problem was about the acceptance or rejection of incoming orders based on the definition of minimum price for the orders and the assignment of vehicles to orders. This multi-objective problem was solved with the ELECTRE III method.

Cavar et al. [10], in 2005 applied AHP to the selection of the best VRP algorithm to be used for a particular case, considering factors like the number of vehicles to use, the time necessary to calculate the routes and the overall travelled distance of the vehicle fleet.

Later, in 2008, an interesting application of MCDA to a routing problem was carried out by Tavana et al. [39], within the subject of Joint Air Operations. Their 
goal was to model a problem that comprises the assignment of aerial vehicles to mission packages. The MCDA model focus on four competing objectives considered for the assessment of vehicle-target allocation, and to solve the problem, the Analytic Hierarchy Process (AHP) method is also used in the model.

In [20] the authors intended to optimize the design of a supply centre for public service, and they employ Fuzzy-AHP to decide on the service facility types and then use VRP solution methods to find the number and location of the facilities. Criterion like delivery level, service level, supply cost, customers response (satisfaction), transportation and service information were used.

Still in 2008, an application of goal programming (GP) methodology to model a single vehicle routing problem with multiple routes was proposed in [21]. The developed model is solved using a heuristic method based on an elementary Shortest Path Algorithm with Resource Constraints.

Later, in 2010, Tavana and Bourgeois [38] focused on the problem of operational planning and navigation of autonomous underwater vehicles. A dynamic multiple criteria support system was developed and the authors employed MCDA methods, along with other methodologies, to assist in mission planning carried out by the United States Navy.

A combination of GP and genetic algorithm was employed in [18] to model and solve a multi-objective VRP with time windows. The considered objectives were the minimization of total required fleet size and minimization of total travelling distance, while constraints such as capacity and time windows are fulfilled.

A more recent work was presented in 2012 by Ries and Ishizaka [29]. The authors developed a DSS to solve a routing problem of Unmanned Aerial Vehicles within the scope of maritime surveillance. They applied MCDA methods such as AHP and PROMETHEE to evaluate the operational scenarios produced by the routing problem algorithm.

Also in 2012, a combination of AHP and TOPSIS was presented in [44] as a solution method for assessing alternative routes for a VRP.

\section{Methodology}

Previously in this paper we stated that our intent was to present a prototype Decision Support System (DSS) to help managers deal with multi-criteria decision-making applied to route planning in the context of waste collection. In our concept, the DSS to be developed shall include three different modules: 1) a route optimization module, 2) a MCDA module, and 3) a waste generation prediction module to predict waste generation rates and determine priority levels of the collection points. The route optimization module has its origin on improvements made to previously presented works within the scope of the GATOP project $[15,16,26,27,28]$. The same is applicable to the waste generation prediction module [17]. The base for the MCDA module development was the beSmart software v1.1 [5]. Improvements were made to the software so it could better meet our purpose with the DSS. In this section we will present the modifications and improvements made to the route optimization module and the MCDA module, as well as the adopted methodologies. 


\subsection{Route Optimization for Waste Collection}

Defining Different Alternatives for Waste Collection Routes. Waste management companies usually have special concern about their waste collection system and how efficient their route planning procedures currently are and how they can be improved. Many companies employ fixed collection routines and schedules, and surely that simplifies resource management. However, performing route optimization according to specific objectives may represent a relevant source of extra revenue, and that is a great motivator for the employment of better management practices, the application of better route planning algorithms and/or decision aid software. From now on in this document, the term solution refers to a set of optimized routes that can be assigned to a vehicle fleet.

The definition of objectives when performing route planning is crucial in order to meet certain goals or quotas for waste collection. For example, one common objective is to minimize the distance travelled by the vehicles while visiting a set collection points, and less mileage means less vehicle maintenance costs and fuel expenses and also less greenhouse gas emissions. For this study, we used a list of important objectives that were agreed with the WMC, that should be taken into consideration while aiming to optimize routes:

- Minimize Total Distance Travelled - MinD

- Maximize Total Collected Quantity - MaxQ

- Maximize Performance - MaxP (quantity collected per kilometre travelled)

- Maximize Number of Ecopoints Collected - MaxE

- Minimize Number of Vehicles Used - MinV

- Maximize Number of Priority Points Collected - MaxPP

Performance Indicators in Waste Collection. The route planning procedure often relies on specific algorithms and/or software tools. The quality of the solutions obtained with those tools and techniques needs to be assessed using some performance indicators. A performance indicator is a type of performance measurement, and in this case it quantifies a certain factor related to waste collection routes. All the values for the performance indicators are quantitative, which makes it easier for comparisons when using some MCDA techniques. These performance indicators are in fact a set of criteria that have influence in the context of route planning for waste collection. The performance indicators considered for this study are the following:

- Total Distance Travelled (per solution)

- Average Distance Travelled (per route)

- Total Collected Quantity (per solution)

- Average Collected Quantity (per route)

- Total Collection Performance (per solution)

- Average Collection Performance (per route)

- Number of Collected Ecopoints (per solution)

- Average Number of Collected Ecopoints (per route) 
- Number of Vehicles used (per solution)

- Number of Priority Points Collected (per solution)

- Average Number of Priority Points Collected (per route)

- Number of High Priority Ecopoints Collected (per solution)

A Cellular Genetic Algorithm to solve the CTOPTW. The final model we used for route planning was the capacitated team orienteering problem with time windows (CTOPTW). As mentioned in a previous section, there are several methodologies capable of dealing with (solving) the TOP and its variants. Nonetheless, we opted to follow a research line we have been pursuing, which is the application of genetic algorithms (GAs) to solve optimization problems, specially routing problems $[15,16,26,27,28]$. More recently, one of our focus has been the employment of celular genetic algorithms (cGAs) since the experimental results achieved with this method performed better than our previously developed GAs. So, in order to solve the CTOPTW, we developed an algorithm based on the cGA methodology. We also made adjustments to the algorithm so it could deal with different objective functions and output optimized routes accordingly.

\section{2 beSmart A Multi-Criteria Decision Aid Software Application}

Software Description and Improvements Made. There are many different MCDA methods, and there are also many tools developed to implement the MCDA methods. In 2010, Seixedo and Tereso [31] have assembled a list of the available MCDA tools and developed a Multi-Criteria Decision Aid Software Application for selecting MCDA software using the Analytic Hierarchy Process (AHP) method. Later, in 2011, improved MCDA software for the same purpose was developed by Tereso et al. [40]. The software tool was named beSmart [5], and was designed to be a general-purpose application, which can load and process data to help solving any MCDA problem. The beSmart became an interesting tool for us to explore and integrate in the DSS prototype we intend to develop.

Although the beSmart software was at a good state of development, some improvements were made in order to offer a better user experience. This was achieved by enhancing the Graphical User Interface (GUI), using better displaying of options and commands in the menu bar, and by improving the contents of tips and instructions displayed to the user throughout the decision-making process. Changes to the solution explorer module were also made, in order to present more useful information on the solutions produced. In addition, the option to perform sensitivity analysis was included in the solution explorer.

MCDA Methods Available in beSmart. There are three MCDA methods embedded within beSmart: 1) SMART, 2) AHP, and 3) ValueFn. The SMART method [14] consists of assigning a score to each alternative, and the higher the scores are, the more importance the alternative represents. In the AHP method [30], a structuring process of the problem occurs so that it is decomposed into a hierarchy of sub-problems. Then, the DM evaluates the relative importance of 
these sub-problems (criteria) by pairwise comparison, where a degree of importance is given to each criterion in relation to another. The AHP method converts these evaluations to numerical values (weights or priorities), which are used to calculate a score for each alternative. A consistency rate measures the extent to which the DM has been consistent in the comparison done. This rate should be lower than 0.10 [30]. In the ValueFn method [9,23], the evaluation of alternatives is directly fitted into a function. It can be a maximization or a minimization function, depending on the DMs intention to maximize or minimize a given attribute. For further information and more detailed explanations on how these MCDA methods were implemented into beSmart, one should check the work in [31] and [40].

\subsection{A Prototype Decision Support System to Improve Waste Collection}

Since each module of the DSS prototype is also a stand-alone software tool, a proper design is needed in order to establish how each module interacts with each other, how information flows between them and how solutions are presented to the end-user. A schematic in figure 1 shows how the DSS's operates. Initially, the waste generation prediction module forecasts the current state of each collection point in terms of quantity of waste stored in its containers. These informations are fed to the route optimization module, which in turn proceeds with the computation of collection routes for all objectives previously presented, although not in a multi-objective approach, with a solution for each objective function being output separately. Once the routes are calculated, their values for each performance indicator (PI) are saved in a text file, which is later loaded in beSmart.

The beSmart software can help to select the best set of routes for specific scenarios of waste collection where the DM needs to weigh the importance of each PI. The decision process occurs during five steps (see figure 2). The first one is the selection of alternatives for comparison, which are sets of routes called solutions. Each solution results from the output of the route optimization module according to a certain objective function. In the next decision step, the DM chooses the PIs he finds relevant for solution assessment. The third step is the definition of weights, and the DM expresses the relative importance of each previously selected PI using either the SMART or AHP method. The DM assigns values to each PI to denote its importance relative to the others. The fourth step is the definition of priorities, and using the AHP or ValueFn methods, one can determine the priority level each PI represents to each solution. Finally, in the fifth step, the comparison results are displayed using charts and ranking lists. It is possibile to perform real-time sensitivity analysis for the selected PIs. This feature enables the DM to examine the trade-offs between a PI and the rest, and also the impact of changing the priority level of each PI individually. Once the decision process in beSmart ends, the DM is presented with a final ranking of alternatives, sorted from the most to the least suitable solution. Using the DSS makes the DM more aware of solution possibilities and their outcomes. 


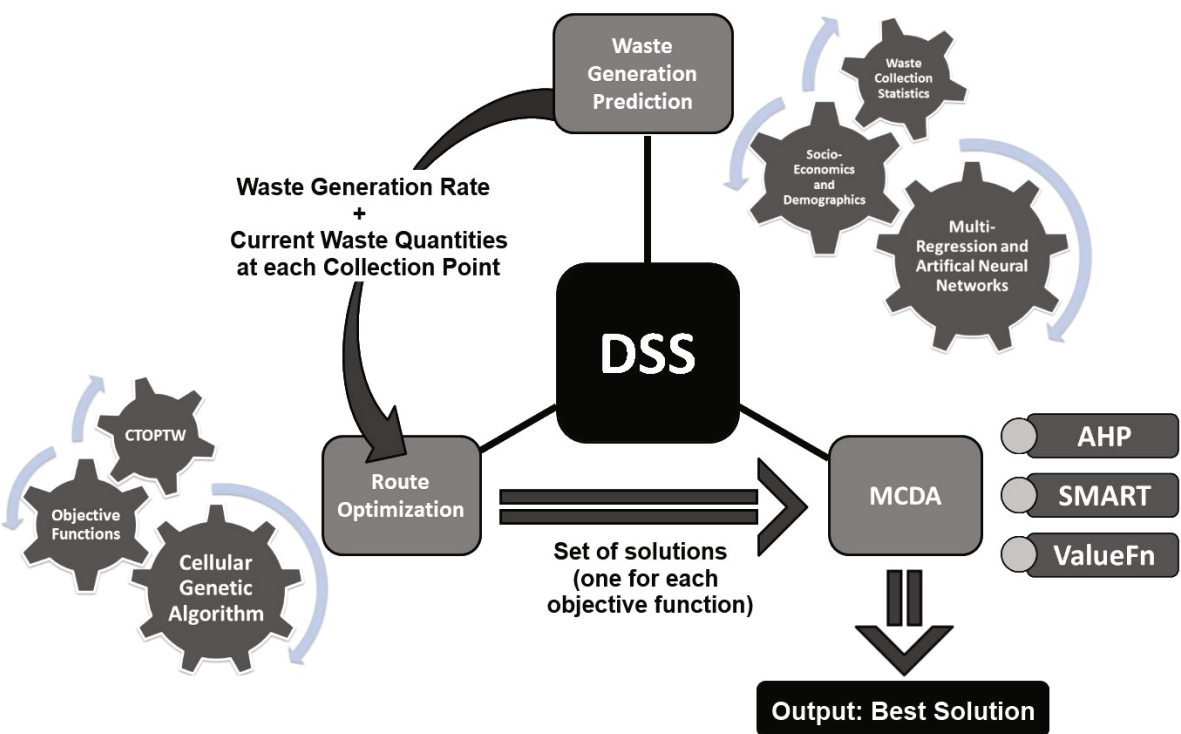

Fig. 1. DSS's Operational Schematic

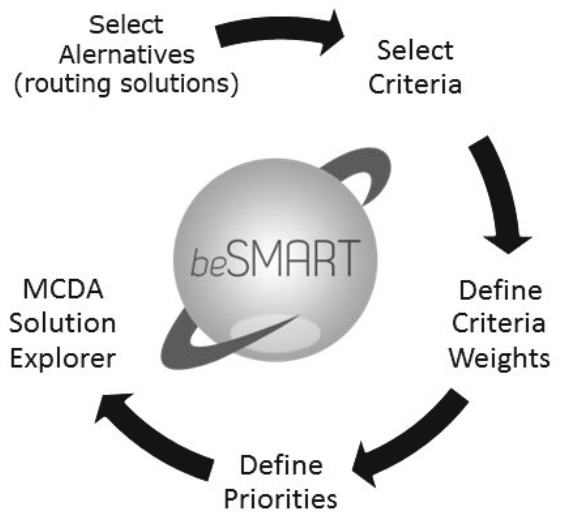

Fig. 2. Steps for Decision-Making in beSmart

\section{$5 \quad$ Experiments and Results}

The assessment of the proposed DSS was done by simulation of real situations of HPW collection, using the WMC's data to design 10 CTOPTW instances. The data sources used to assemble the instances consisted of a list of 94 ecopoints and their GPS coordinates. These 94 ecopoints represent a whole municipality. 
Informations such as the values for time windows and waste quantities at each ecopoint were obtained using the waste generation prediction module of the DSS. Real distances between ecopoints were obtained using Google Maps, a web mapping service application. We made the instances and the test results available for download at "http://pessoais.dps.uminho.pt/zan/GATOP/instEMO2015.zip". With those instances we aimed to prove the usefulness of the DSS and its capabilities to attend waste collection management goals. For each CTOPTW instance, collection routes were planned for three vehicles with the same capacity. The route optimization module assembled sets of three routes for each objective function separately (not multi-objective optimization). However, some global constraints were applied for the cGA to deal with: maximum route duration, maximum distance to travel per route, minimum waste quantity to collect per route, and minimum quantity to collect per solution. The cGA was run 5 times for each of the six objective functions, hence outputting 30 different solution alternatives and the respective values for each PI. The best alternative for each objective function was loaded into beSmart, where the decision-making process took place, with the $12 \mathrm{PIs}$ previously stated used as criteria. We opted to test a combination of MCDA methods during the decision process using beSmart. First we employed the SMART method for the definition of weights, and later, for the definition of priorities, the choice relied on a mixed application of AHP and ValueFn, choosing the method that seemed most appropriate for each criteria. In figure 3, the solution achieved using beSmart for one tested instance is presented. On the upper-left area, the final priority ranking of alternatives is shown. On the bottom-left area, a sensitivity analysis component is available. On the bottom-right a detailed bar chart shows how the weight of each criterion

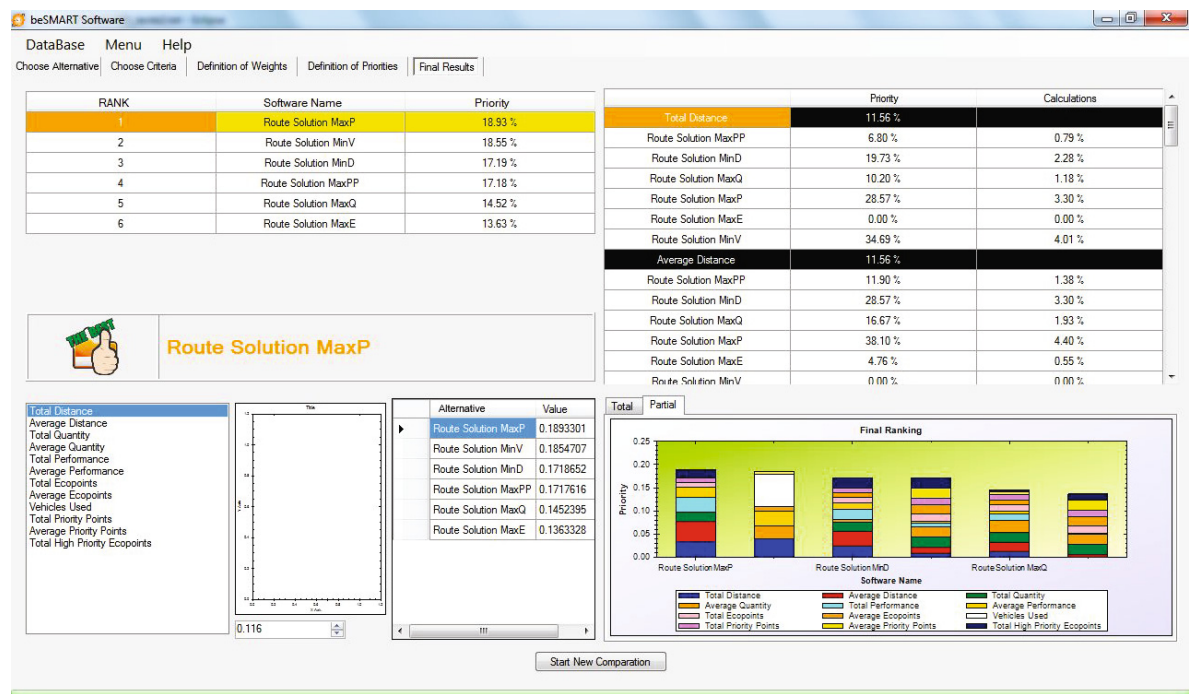

Fig. 3. Results with beSmart for the simulation scenario 
contributed for the ranking of each alternative. Although the best alternative found was "Route Solution MaxP", if the DM expresses different preferences, alternative solutions can be found. Throughout several similar tests, we observed that the DSS has proven its usefulness, as it allows the user to solve waste collection problems, and is able to help analyse the influence of several criteria, weighing their contribution while aiming to attend certain performance levels.

\section{Conclusions and Future Work}

In this work we presented a decision support system (DSS) that provides solutions for problems that arise in waste collection management. One main issue is to perform route planning for selective collection of recyclable waste. We modelled this routing problem as a capacitated team orienteering problem with time windows (CTOPTW), and we developed a cellular genetic algorithm (cGA) to produce solutions. The cGA is able to deal with different objective functions. We presented twelve criteria based on waste collection performance indicators (PIs), and we employed multi-criteria decision analysis (MCDA) methods to assist in decision-making when weighing the importance of each PI in route planning.

The developed DSS includes three modules: waste generation prediction, route optimization, and MCDA. We presented a design for the DSS and assembled a prototype to run experiments at this stage of development. We performed simulations of real problems faced by a waste management company (WMC) responsible for collecting waste for recycling. The DSS was tested with real instances of the problem. In our best knowledge, there are no similar approaches to the one we presented in this paper for dealing in an unified way with several problems related to waste collection management, and so, direct comparisons with alternative systems were not possible. Nevertheless, we assessed the DSS's capabilities in terms of route planning and optimization, with a focus on MCDA. The DSS provides different optimization options and can present alternative solutions for the same instance of HPW collection, depending on the preferences of decision-makers (DMs). These features are advantageous, since the DM's preferences play a central role in selecting the best routes for a certain planning period, according to the WMC's logistic strategy and the importance given to each PI. We obtained positive feedback from the WMC and more improvements are foreseen. We are positive that our DSS design can be of great assistance in the context of waste collection, and WMCs would be able to improve their performances by exploring better collection routines and by adapting to challenges that may arise before them. Overall, the interpretation of computational results output by the DSS can provide meaningful information to waste collection management practitioners. However, it is not advisable to apply the DSS on a daily basis, as it would be an impractical and time-consuming task to go through the decision process every day. Instead, the DSS should be used to outline the WMC's logistic strategy for a longer period such as a week, month or trimester. 
For future work, we intend to simplify the user's interaction with the DSS to enhance overall user experience and accelerate work flow. More experiments with the DSS shall be conducted in more real-case situations to fully validate our approach.

Acknowledgments. This work has been supported by FCT Fundação para a Ciência e Tecnologia within the Project Scope: PEst-OE/EEI/UI0319/2014.

\section{References}

1. Alba, E., Dorronsoro, B.: Solving the vehicle routing problem by using cellular genetic algorithms. In: Gottlieb, J., Raidl, G.R. (eds.) EvoCOP 2004. LNCS, vol. 3004, pp. 11-20. Springer, Heidelberg (2004)

2. Archetti, C., Bianchessi, N., Speranza, M.G.: The capacitated team orienteering problem with incomplete service. Optimization Letters 7(7), 1405-1417 (2013)

3. Archetti, C., Feillet, D., Hertz, A., Speranza, M.G.: The capacitated team orienteering and profitable tour problems. Journal of the Operational Research Society 60, 831-842 (2009)

4. Archetti, C., Hertz, A., Speranza, M.G.: Metaheuristics for the team orienteering problem. Journal of Heuristics 13, 49-76 (2007)

5. BeSmart software development webpage. https://code.google.com/p/besmart/

6. Bouly, H., Dang, D.-C., Moukrim, A.: A memetic algorithm for the team orienteering problem. In: Giacobini, M., Brabazon, A., Cagnoni, S., Di Caro, G.A., Drechsler, R., Ekárt, A., Esparcia-Alcázar, A.I., Farooq, M., Fink, A., McCormack, J., O'Neill, M., Romero, J., Rothlauf, F., Squillero, G., Uyar, A.Ş., Yang, S. (eds.) EvoWorkshops 2008. LNCS, vol. 4974, pp. 649-658. Springer, Heidelberg (2008)

7. Boussier, S., Feillet, D., Gendreau, M.: An exact algorithm for team orienteering problems. 4OR 5, 211-230 (2007)

8. Butt, S.E., Cavalier, T.M.: A heuristic for the multiple tour maximum collection problem. Computers and Operations Research 21, 101-111 (1994)

9. Canada, J.R., Sullivan, W.G.: Economic and Multiattribute Evaluation of Advanced Manufacturing Systems. In: Multiattribute Decision Analysis: utility models. ch. 9. Prentice Hall College Div. (1989)

10. Cavar, I., Gold, H., Caric, T.: Assessment of heuristic algorithms for solving real capacitated vehicle routing problems by analytic hierarchy process. In: 12th World Congress in Intelligent Transport Systems, San Francisco (2005)

11. Chao, I., Golden, B.L., Wasil, E.A.: The team orienteering problem. European Journal of Operational Research 88, 464-474 (1996)

12. Dang, D.-C., Guibadj, R.N., Moukrim, A.: A PSO-based memetic algorithm for the team orienteering problem. In: Di Chio, C., Brabazon, A., Di Caro, G.A., Drechsler, R., Farooq, M., Grahl, J., Greenfield, G., Prins, C., Romero, J., Squillero, G., Tarantino, E., Tettamanzi, A.G.B., Urquhart, N., Uyar, A.Ş. (eds.) EvoApplications 2011, Part II. LNCS, vol. 6625, pp. 471-480. Springer, Heidelberg (2011)

13. Dantzig, G.B., Ramser, J.H.: The Truck Dispatching Problem. Management Science 6(1), 80-91 (1959)

14. Doran, G.T.: There's a S.M.A.R.T. way to write management's goals and objectives. Management Review 70(11), 35-36 (1981) 
15. Ferreira J., Oliveira J., Pereira G., Dias L., Vieira F., Macedo J., Carção T., Leite T., Murta D.: Developing tools for the team orienteering problem - a simple genetic algorithm. In: Proceedings of the 2nd International Conference on Operations Research and Enterprise Systems, Barcelona, pp. 134-140 (2013)

16. Ferreira, J., Quintas, A., Oliveira, J.A., Pereira, G., Dias, L.: Solving the team orienteering problem - developing a solution tool using a genetic algorithm approach. In: Snášel, V., Krömer, P., Köppen, M., Schaefer, G. (eds.) Soft Computing in Industrial Applications. AISC, vol. 223, pp. 365-375. Springer, Heidelberg (2014)

17. Ferreira, J.A., Figueiredo, M.C., Oliveira, J.A.: Forecasting household packaging waste generation: a case study. In: Murgante, B., Misra, S., Rocha, A.M.A.C., Torre, C., Rocha, J.G., Falcão, M.I., Taniar, D., Apduhan, B.O., Gervasi, O. (eds.) ICCSA 2014, Part III. LNCS, vol. 8581, pp. 523-538. Springer, Heidelberg (2014)

18. Ghoseiri, K., Ghannadpour, S.F.: Multi-objective vehicle routing problem with time windows using goal programming and genetic algorithm. Applied Soft Computing 10(4), 1096-1107 (2010)

19. Gomes, A., Matos, M., Carvalho, I.: Separate collection of the biodegradable fraction of MSW: An economic assessment. Waste Manage 28, 1711-1719 (2008)

20. Hwang, H., Choi, B., Lee, K., Cho, G.: Supply Center Planning Model Using Fuzzy-AHP and VRP. In: 3rd International Conference on Innovative Computing Information and Control - ICICIC 2008, p. 109 (2008)

21. Jolai, F., Aghdaghi, M.: A Goal Programming Model for Single Vehicle Routing Problem with Multiple Routes. Journal of Industrial and Systems Engineering 2(2), 154-163 (2008)

22. Ke, L., Archetti, C., Feng, Z.: Ants can solve the team orienteering problem. Computers and Industrial Engineering 54, 648-665 (2008)

23. Keeney, R., Raiffa, H.: Decision with Multiple Objectives: preferences and value tradeoffs. Cambridge University Press (1993)

24. Labadi, N., Mansini, R., Melechovský, J., Calvo, R.W.: The Team Orienteering Problem with Time Windows: An LP-based Granular Variable Neighborhood Search. European Journal of Operational Research 220(1), 15-27 (2012)

25. Montemanni, R., Weyland, D., Gambardella, L.M.: An enhanced ant colony system for the team orienteering problem with time windows. In: International Symposium on Computer Science and Society (ISCCS), pp. 381-384 (2011)

26. Mota, G., Abreu, M., Quintas, A., Ferreira, J., Dias, L.S., Pereira, G.A.B., Oliveira, J.A.: A genetic algorithm for the TOPdTW at operating rooms. In: Murgante, B., Misra, S., Carlini, M., Torre, C.M., Nguyen, H.-Q., Taniar, D., Apduhan, B.O., Gervasi, O. (eds.) ICCSA 2013, Part I. LNCS, vol. 7971, pp. 304-317. Springer, Heidelberg (2013)

27. Oliveira, J.A., Mota, G., Ferreira, J., Figueiredo, M., Dias, L., Pereira, G.: A decision support system for waste collection modeled as TOPTW variant. In: WASTES: Solutions, Treatments and Opportunities - 2nd International Conference (accepted, 2013)

28. Oliveira, J.A., Ferreira, J., Figueiredo, M., Dias, L., Pereira, G.: Comparação de dois algoritmos genéticos aplicados ao TOP. XI Congreso Galego de Estatística e Investigación de Operacións (accepted, 2013)

29. Ries, J., Ishizaka, A.: A multi-criteria support system for dynamic aerial vehicle routing problems. In: Proceedings of IEEE 2nd International Conference on Communications, Computing and Control Applications (CCCA), pp. 1-4 (2012)

30. Saaty, T.L.: The Analytic Hierarchy Process. McGraw-Hill, New York (1980) 
31. Seixedo, C., Tereso, A.: A Multicriteria Decision Aid Software Application for selecting MCDA Software using AHP. In: 2nd International Conference on Engineering Optimization, Lisbon, Portugal (2010)

32. Sevkli, A.Z., Sevilgen, F.E.: Discrete particle swarm optimization for the team orienteering problem. Turk. Journal Elec. Eng. \& Comp. Sci. 20(2) (2012)

33. Souffriau, W., Vansteenwegen, P., Van Oudheusden, D.: A Path Relinking Approach for the Team Orienteering Problem. Computers \& Operations Research, Metaheuristics for Logistics and Vehicle Routing 37(11), 1853-1859 (2010)

34. Souffriau, W., Vansteenwegen, P., Berghe, G.V., Oudheusden, D.V.: The Multiconstraint Team Orienteering Problem with Multiple Time Windows. Transportation Science 47(1), 53-63 (2013)

35. Tang, H., Miller-Hooks, E.: A TABU search heuristic for the team orienteering problem. Computers \& Operations Research 32, 1379-1407 (2005)

36. Tasgetiren, M.F.: A Genetic Algorithm with an Adaptive Penalty Function for the Orienteering Problem. Journal of Economic and Social Research 4(2), 1-26 (2002)

37. Tasgetiren, M.F., Smith, A.E.: A genetic algorithm for the orienteering problem. In: Proceedings of the 2000 Congress on Evolutionary Computation (CEC2000), vol. 2, pp. 910-915 (2000)

38. Tavana, M., Bourgeois, B.S.: A multiple criteria decision support system for autonomous underwater vehicle mission planning and control. International Journal of Operational Research 7(2), 216-239 (2010)

39. Tavana, M., Bailey, M.D., Busch, T.E.: A multi-criteria vehicle-target allocation assessment model for network-centric Joint Air Operations. International Journal of Operational Research 3(3) (2008)

40. Tereso, A., Sampaio, A., Frade, H., Costa, M., Abreu, T.: beSMART: a software tool to support the selection of decision software. In: International Conference on Engineering UBI2011 (ICEUBI2011), Covilhã, Portugal (2011)

41. Tricoire, F., Romauch, M., Doerner, K.F., Hartl, R.F.: Heuristics for the multiperiod orienteering problem with multiple time windows. Computers \& Operations Research 37, 351-367 (2010)

42. Vansteenwegen, P., Souffriau, W., Van Oudheusden, D.: Iterated local search for the team orienteering problem with time windows. Computers \& Operations Research 36, 3281-3290 (2009)

43. Vansteewegen, P., Souffriau, W., Van Oudheusden, D.: A guided local search metaheuristic for the team orienteering problem. European Journal of Operational Research 196(1), 118-127 (2009)

44. Yilmaz Z., Aplak, H.S., Vehicle routing by revaluing the alternative routes by using AHP-TOPSIS Combination. In: Proceedings of X International Logistics \& Supply Chain Congress, İstanbul, Turkey, pp. 304-310 (2012)

45. Zak, J.: The MCDA methodology applied to solve complex transportation decision problems. In: Proceedings of the 13th Mini Euro Conference, Bari, Italy (2002) 